\title{
Scalar diffraction field calculation from curved surfaces via Gaussian beam decomposition
}

\author{
Erdem Şahin* and Levent Onural \\ Department of Electrical and Electronics Engineering, Bilkent University, TR-0680o Bilkent, Ankara, Turkey \\ *Corresponding author: sahin@ee.bilkent.edu.tr
}

Received March 9, 2012; accepted April 26, 2012;

posted May 1, 2012 (Doc. ID 164499); published June 29, 2012

\begin{abstract}
We introduce a local signal decomposition method for the analysis of three-dimensional (3D) diffraction fields involving curved surfaces. We decompose a given field on a two-dimensional curved surface into a sum of properly shifted and modulated Gaussian-shaped elementary signals. Then we write the 3D diffraction field as a sum of Gaussian beams, each of which corresponds to a modulated Gaussian window function on the curved surface. The Gaussian beams are propagated according to a derived approximate expression that is based on the RayleighSommerfeld diffraction model. We assume that the given curved surface is smooth enough that the Gaussian window functions on it can be treated as written on planar patches. For the surfaces that satisfy this assumption, the simulation results show that the proposed method produces quite accurate 3D field solutions. C 2012 Optical Society of America

OCIS codes: $\quad 070.7345,090.0090,090.1760,090.1995$
\end{abstract}

\section{INTRODUCTION}

Recording digital holograms on curved surfaces and reconstruction from such curved holograms are attractive alternatives to conventional planar geometries. Spherical geometries are shown to be more useful compared to planar ones to reduce the resolution requirements of the sensor and display devices [1,2]. In the work of Hahn et al. [3] , a curved array of spatial light modulators (SLMs) is used as a holographic display device to increase the field of view compared to the conventional planar array of SLMs. Similar possible advantages of the curved display and sensor devices for holography indicate the need for the theoretical analysis of diffraction between curved surfaces. In this paper the local Gaussian beam decomposition method is proposed to calculate the diffraction field from such curved surfaces.

The interest of graphics and optics communities to computer-generated holography has been significant, such that several methods are proposed to generate digital holograms of three-dimensional (3D) objects $[\underline{4}, \underline{5}]$. For the simple case of planar objects, the Rayleigh-Sommerfeld (RS) formulation and its paraxial approximation (Fresnel model) can be used to find the desired $3 \mathrm{D}$ field [ $\underline{6}, 7]$. For the more general case of 3D objects, having depth, the $3 \mathrm{D}$ object is commonly modeled as a collection of self-illuminating point sources [ $\underline{8}-11]$. The field due to a single point source is found by the RS or Fresnel diffraction model. The 3D diffraction field due to the object is then found by superposing the fields due to the point light sources. In some applications, the object is assumed to be formed by planar patches [12-14]. In this case, the plane wave decomposition (PWD) method (angular spectrum) is applied to each patch to calculate the $3 \mathrm{D}$ diffraction field. Note that the PWD method is the frequency domain counterpart of the RS integral [15].

For the problem of finding the $3 \mathrm{D}$ field due to a field specified on a curved surface (or an object), the commonly used source model approaches [ㅁ-14] do not produce accurate results, because they ignore the mutual couplings between the field samples on the given curved surface. In other words, if the $3 \mathrm{D}$ field is calculated by using such source model approaches, then the subsequently reconstructed field on the same curved surface is found to be inconsistent with the original one. The mutual coupling problem is solved by using a field model approach, based on PWD, in [16, 17] , resulting in an exact method to calculate the diffraction field from a given curved surface. However, for large sizes of curved surfaces, this method becomes impractical because of an intolerable increase in the computational complexity and the related memory requirement.

Instead of global plane waves, we propose to use local Gaussian beams to find the diffraction field from curved surfaces. For the case of a planar input surface, the decomposition of a two-dimensional (2D) signal as a sum of Gaussian elementary signals was suggested by Gabor [18]. After that, several studies, which aim to find Gabor's expansion coefficients and expand an optical signal into a set of Gaussian beams, related to this decomposition were introduced by Bastiaans $[\underline{19}, \underline{20}]$. In those works the input surface is restricted to be planar, and an explicit 3D Gaussian beam decomposition expression is not given. In this paper, we study the more general case of curved input surfaces. Furthermore, for the propagation of 3D Gaussian beams, we develop an approximate explicit expression that is based on the RS diffraction model. Such an expression enables us to use an optically valid model for the propagation of nonparaxial Gaussian beams.

In Section 2, we give a brief explanation of the global PWD and the method proposed in [16] related to PWD. Then we introduce the local Gaussian beam decomposition method in Section 3. We test our approach and present the 
simulation results in Section $\underline{4}$. Finally, we draw conclusions in Section $\underline{5}$.

\section{PLANE WAVE DECOMPOSITION}

A possible way to calculate the $3 \mathrm{D}$ diffraction field due to a field specified on a planar surface is to use the PWD. The PWD is a global signal decomposition method where the basis functions are the infinite extent plane waves [6]. That is, a monochromatic 3D field that can be formed by propagating waves can be written as a sum of infinite extent plane waves propagating toward different directions:

$$
u(x, y, z)=\iint_{B} A\left(f_{x}, f_{y}\right) \exp \left\{j 2 \pi\left(f_{x} x+f_{y} y+f_{z} z\right)\right\} \mathrm{d} f_{x} \mathrm{~d} f_{y},
$$

where $f_{x}^{2}+f_{y}^{2}+f_{z}^{2}=1 / \lambda^{2}$ and $\lambda$ is the wavelength of the plane waves. (Please note that units of all spatial frequencies are cycles per unit length.) Here we exclude the evanescent waves and take into account only the propagating plane waves whose frequency components along the $x$ and $y$ axes satisfy $f_{x}^{2}+f_{y}^{2} \leq \frac{1}{\lambda^{2}}$. Thus, the integration is over a circular frequency band, which we call $B$, having a radius $\frac{1}{\lambda}$. In this paper, we assume that a given $3 \mathrm{D}$ field $u(x, y, z): \mathbb{R}^{3} \rightarrow \mathbb{C}$ is formed by a superposition of propagating plane waves with $f_{z}>0$; in other words, the component of the propagation direction along the $z$ axis is always positive [21]. The coefficient of each plane wave is found by an integration over the spatial domain as

$$
A\left(f_{x}, f_{y}\right)=\int_{-\infty}^{\infty} \int_{-\infty}^{\infty} u(x, y, 0) \exp \left\{-j 2 \pi\left(f_{x} x+f_{y} y\right)\right\} \mathrm{d} x \mathrm{~d} y
$$

Note that these coefficients correspond to the Fourier transform of the $2 \mathrm{D}$ input field $u(x, y, 0)$ given on the $z=0$ plane, where the Fourier transform of a 2D function $a(x, y)$, from the $(x, y)$ domain to $\left(f_{x}, f_{y}\right)$ domain, is defined as

$$
A\left(f_{x}, f_{y}\right)=\int_{-\infty}^{\infty} \int_{-\infty}^{\infty} a(x, y) \exp \left\{-j 2 \pi\left(f_{x} x+f_{y} y\right)\right\} \mathrm{d} x \mathrm{~d} y
$$

The PWD is also used to calculate the 3D field due to a field specified on a curved surface $[16,17]$. In the discrete case, the decomposition is formulated as a matrix vector product

$$
\Psi=\Phi a
$$

In this equation, each column of the matrix $\Phi$ represents the field samples that a particular plane wave produces at the discrete points on a given curved surface and $\Psi$ is the array of the given field samples at the same discrete points of the curved surface. $\boldsymbol{a}$ is the array of coefficients of the plane waves to be found. Once the coefficients of the plane waves are found, the $3 \mathrm{D}$ diffraction field is written as a sum of plane waves, each of which is weighted by its corresponding coefficient. Note that, as opposed to the planar input surface case, the columns of the matrix $\Phi$ are not orthogonal for nonplanar surfaces. As a result, the computational complexity in calculating the coefficients increases significantly $[\underline{16}, 17]$.

\section{LOCAL BEAM DECOMPOSITION}

We start with developing formulations for the calculation of the diffraction field due to a field specified on a planar input surface. This will relieve us from dealing with the structure of the surface. We will take into account the curvature of the input surface in Subsection 3.B and extend the formulations developed for planar input surfaces.

\section{A. Decomposition for the Planar Input Surface Case}

Instead of writing the 3D diffraction field as a sum of infinite extent plane waves by using PWD, we want to write it as a sum of local beams. We define a local beam in $3 \mathrm{D}$ space as the beam that corresponds to a shifted and modulated version of a local function on the given input surface. Hence, we start with writing the field $u(x, y, 0)$ specified on a planar surface at $z=0$ in the form of a continuous short time Fourier decomposition as [22]

$$
\begin{aligned}
u(x, y, 0)= & \iiint \int a\left(\xi, \eta, f_{x}, f_{y}\right) g(x-\xi, y-\eta) \\
& \times \exp \left\{j 2 \pi\left(f_{x} x+f_{y} y\right)\right\} \mathrm{d} \xi \mathrm{d} \eta \mathrm{d} f_{x} \mathrm{~d} f_{y},
\end{aligned}
$$

where $g(x, y)$ is a unit energy local synthesis window function. The corresponding analysis equation of Eq. (5) is given as

$$
\begin{aligned}
a\left(\xi, \eta, f_{x}, f_{y}\right)= & \iint u(x, y, 0) g^{*}(x-\xi, y-\eta) \\
& \times \exp \left\{-j 2 \pi\left(f_{x} x+f_{y} y\right)\right\} \mathrm{d} x \mathrm{~d} y .
\end{aligned}
$$

Equation $\underline{5}$ can be viewed as a decomposition of the input signal $u(x, y, 0)$ into a sum of modulated and shifted versions of the synthesis window function $g(x, y)$. The discrete version of Eq. $\underline{5}$ is

$$
\begin{aligned}
u(x, y, 0)= & \sum_{m} \sum_{n} \sum_{k} \sum_{l} a_{m n k l} g(x-m X, y-n Y) \\
& \times \exp \left\{j 2 \pi\left(k F_{x} x+l F_{y} y\right)\right\}
\end{aligned}
$$

where $X, Y$ are the shift steps in space and $F_{x}, F_{y}$ are the shift steps in frequency. In the discrete case, the corresponding analysis window function is not same as the synthesis window function. One can find an analysis window function $w(x, y)$ corresponding to the synthesis window function $g(x, y)$ such that the signal $u(x, y, 0)$ is obtained exactly with the coefficients $a_{m n k l}[\underline{19}, 23]$. Thus,

$$
\begin{aligned}
a_{m n k l}= & \iint u(x, y, 0) w^{*}(x-m X, y-n Y) \\
& \times \exp \left\{-j 2 \pi\left(k F_{x} x+l F_{y} y\right)\right\} \mathrm{d} x \mathrm{~d} y .
\end{aligned}
$$

As a special case of the decomposition given by Eq. (ㄱ), Gabor considers the Gaussian-shaped signal as the synthesis window function [18]. Each modulated and shifted version of a Gaussian-shaped elementary signal occupies the smallest possible area in the space-frequency domain. This is a particularly desirable property for our aims in this paper. In [18], the decomposition is restricted to the case of critical sampling $\left(X F_{x}=1, Y F_{y}=1\right)$ of the space-frequency domain. However, for the choice of Gaussian-shaped signal as the synthesis 
window function, it is shown in 24 that oversampling $\left(X F_{x}<1, Y F_{y}<1\right)$ should be preferred, for a numerically stable reconstruction.

The Gaussian signal decomposition on the input surface corresponds to the Gaussian beam decomposition in the 3D space. Let us define $g_{h}\left(\boldsymbol{x}-\boldsymbol{r}, f_{x}, f_{y}\right)$ as the 3D field expression of the Gaussian beam at the observation point $x=[x, y, z]$ due to the modulated Gaussian window function, at $\boldsymbol{r}=[\xi, \eta, 0]^{T}$ on the $z=0$ plane, with the modulation frequencies $f_{x}$ and $f_{y}$ along the $x$ and $y$ axes, respectively. The 3D field at $x$ due to the field $u(x, y, 0)$ specified on $z=0$ plane is then written as an integration over Gaussian beams by using the decomposition given by Eq. (5)

$$
u(\boldsymbol{x})=\iiint \int a\left(\xi, \eta, f_{x}, f_{y}\right) g_{h}\left(\boldsymbol{x}-\boldsymbol{r}, f_{x}, f_{y}\right) \mathrm{d} \xi \mathrm{d} \eta \mathrm{d} f_{x} \mathrm{~d} f_{y} .
$$

Now, let us find an explicit expression for $g_{h}\left(\boldsymbol{x}-\boldsymbol{r}, f_{x}, f_{y}\right)$. We know that the beams having higher propagation angles with respect to the direction of the surface normal correspond to the modulated Gaussian window functions with higher modulation frequencies on the input plane. Hence, the range of propagation directions of the beams with respect to the direction of the surface normal depends on the frequency content of the signal being analyzed. Because of this, as the bandwidth of the signal increases the propagating beams are no longer paraxial. Although the accurate RS model can be successfully used for such nonparaxial cases, it is difficult to obtain an explicit expression for Gaussian beams under the RS model (to our knowledge there is no explicit expression of the Gaussian beam obtained under the RS model in the literature). Therefore, we propose an approximate formula that is still based on the RS model. For an input field $u_{0}(x, y)=u(x, y, 0)$ given on the $z=0$ plane, we find the diffraction field over a planar surface, located at a distance $z$ and parallel to the input plane, as

$$
u_{z}(x, y) \approx h_{z}(x, y) U_{0}\left(\frac{x}{\lambda \sqrt{x^{2}+y^{2}+z^{2}}}, \frac{y}{\lambda \sqrt{x^{2}+y^{2}+z^{2}}}\right) .
$$

This expression produces quite accurate results even for nonparaxial cases, as long as the observation distance $z$ is sufficiently large and the given input field $u_{0}(x, y)$ has a sufficiently narrow support around $(x, y)=(0,0)$. In Eq. $(\underline{10})$

$$
h_{z}(x, y)=\frac{z}{j \lambda} \frac{\exp \left\{j \frac{2 \pi}{\lambda} \sqrt{x^{2}+y^{2}+z^{2}}\right\}}{x^{2}+y^{2}+z^{2}}
$$

is the 2D RS kernel; $x /\left(\lambda \sqrt{x^{2}+y^{2}+z^{2}}\right)$ and $y /\left(\lambda \sqrt{x^{2}+y^{2}+z^{2}}\right)$ are the instantaneous frequencies of the RS kernel at $(x, y)$ along the $x$ and $y$ directions, respectively; and $U_{0}\left(f_{x}, f_{y}\right)$ is the Fourier transform of the given input field $u_{0}(x, y)$. By the way, because we restrict the 3D field only to superposition of propagating waves (i.e., evanescent components are zero), $U_{0}\left(f_{x}, f_{y}\right)=0$ if $\left(f_{x}, f_{y}\right) \notin B$, where $B$ represents the circular frequency band having a radius $\frac{1}{\lambda}$, as before. The expression given by Eq. (10) is applicable for a sufficiently large observation distance $z$, because in this case the RS kernel can be successfully approximated locally as a single frequency complex exponential. For example, the diffraction field at $z=0.1 \mathrm{~mm}$ due to a Gaussian window function on $z=0$ plane having a parameter of $10^{-5} \mathrm{~m}$ (typical case in our experiments) is calculated by the approximate expression with a normalized error in the order of $10^{-2}$. However, for $z>0.1 \mathrm{~m}$ the normalized error is found to be in the order of $10^{-8}$. Note that we define the normalized error, in approximating a signal $f(x, y)$ as $\tilde{f}(x, y)$, as

$$
1-\frac{|\langle f(x, y), \tilde{f}(x, y)\rangle|^{2}}{\langle f(x, y), f(x, y)\rangle\langle\tilde{f}(x, y), \tilde{f}(x, y)\rangle},
$$

where $\left\langle f_{1}(x, y), f_{2}(x, y)\right\rangle$ is the inner product of $f_{1}(x, y)$ and $f_{2}(x, y)$. We will use the defined normalized error as the error measure in the simulations.

In order to write the Gaussian beam decomposition by using Eq. (10), we need the Fourier domain expressions of the modulated and shifted Gaussian window functions. The Fourier transform of a Gaussian window function $g(x, y)=c \exp \left\{\left(x^{2}+y^{2}\right) / \sigma^{2}\right\}$ is given as [25], $\mathcal{F}\{g(x, y)\}=$ $c \sigma^{2} \pi \exp \left\{-\pi^{2} \sigma^{2}\left(f_{x}^{2}+f_{y}^{2}\right)\right\}$, where $\mathcal{F}$ denotes the Fourier transform from the $(x, y)$ domain to $\left(f_{x}, f_{y}\right)$ domain as defined by Eq. (3) and $c$ is a constant making $g(x, y)$ a unit energy function. The Fourier transform of a shifted Gaussian window function is then written as $\mathcal{F}\{g(x-\xi, y-\eta)\}=$ $c \sigma^{2} \pi \exp \left\{-\pi^{2} \sigma^{2}\left(f_{x}^{2}+f_{y}^{2}\right)\right\} \exp \left\{-j 2 \pi\left(f_{x} \xi+f_{y} \eta\right)\right\}$. Finally, the Fourier transform of a shifted and modulated Gaussian window function is obtained as

$$
\mathcal{F}\{g(x-\xi, y-\eta) \exp (j 2 \pi \mu x) \exp (j 2 \pi \nu y)\}=c \sigma^{2} \pi \exp \left\{-\pi^{2} \sigma^{2}\left[\left(f_{x}-\mu\right)^{2}+\left(f_{y}-\nu\right)^{2}\right]\right\} \exp \left\{-j 2 \pi\left[\left(f_{x}-\mu\right) \xi+\left(f_{y}-\nu\right) \eta\right]\right\}
$$

Hence, $g_{h}\left(\boldsymbol{x}-\boldsymbol{r}, f_{x}, f_{y}\right)$ is found by substituting Eq. (13) in Eq. (10) as

$$
\begin{aligned}
g_{h}\left(\boldsymbol{x}-\boldsymbol{r}, f_{x}, f_{y}\right) \approx & h_{z}(x-\xi, y-\eta) \\
& \times c \sigma^{2} \pi \exp \left\{-\pi^{2} \sigma^{2}\left[\left(\frac{x-\xi}{\lambda \sqrt{(x-\xi)^{2}+(y-\eta)^{2}+z^{2}}}-f_{x}\right)^{2}+\left(\frac{y-\eta}{\lambda \sqrt{(x-\xi)^{2}+(y-\eta)^{2}+z^{2}}}-f_{y}\right)^{2}\right]\right\} \\
& \times \exp \left\{-j 2 \pi\left[\left(\frac{x-\xi}{\lambda \sqrt{(x-\xi)^{2}+(y-\eta)^{2}+z^{2}}}-f_{x}\right) \xi+\left(\frac{y-\eta}{\lambda \sqrt{(x-\xi)^{2}+(y-\eta)^{2}+z^{2}}}-f_{y}\right) \eta\right]\right\}
\end{aligned}
$$


The 3D field for a function $u_{0}(x, y)$ on $z=0$ plane is then found by substituting $g_{h}\left(\boldsymbol{x}-\boldsymbol{r}, f_{x}, f_{y}\right)$ given by Eq. (14) into Eq. (9) as

$$
\begin{aligned}
u(\boldsymbol{x}) \approx & \iiint \int a\left(\xi, \eta, f_{x}, f_{y}\right) h_{z}(x-\xi, y-\eta) \\
& \times c \sigma^{2} \pi \exp \left\{-\pi^{2} \sigma^{2}\left[\left(\frac{x-\xi}{\lambda \sqrt{(x-\xi)^{2}+(y-\eta)^{2}+z^{2}}}-f_{x}\right)^{2}+\left(\frac{y-\eta}{\lambda \sqrt{(x-\xi)^{2}+(y-\eta)^{2}+z^{2}}}-f_{y}\right)^{2}\right]\right\} \\
& \times \exp \left\{-j 2 \pi\left[\left(\frac{x-\xi}{\lambda \sqrt{(x-\xi)^{2}+(y-\eta)^{2}+z^{2}}}-f_{x}\right) \xi+\left(\frac{y-\eta}{\lambda \sqrt{(x-\xi)^{2}+(y-\eta)^{2}+z^{2}}}-f_{y}\right) \eta\right]\right\} \mathrm{d} \xi \mathrm{d} \eta \mathrm{d} f_{x} \mathrm{~d} f_{y} .
\end{aligned}
$$

At this point we should note that, as mentioned before, the accuracy of the 3D Gaussian beam expressions used in Eq. (15) is better for a narrower Gaussian window function. Therefore, in order to calculate the 3D field with an acceptable accuracy by using Eq. (15), a sufficiently narrow Gaussian window function should be chosen.

The discrete version of Eq. (15) is obtained by sampling the space-frequency domain, at $\xi=m X, \eta=n Y$, and $f_{x}=k F_{x}$, $f_{y}=l F_{y}$ as assumption of $S$ enables us to represent a given field $u(r)$, on $S$, locally on the tangent planes of $S$. Therefore, in order to decompose $u(r)$ as a sum of modulated Gaussian window functions on the tangent planes of $S$, we first define the Gaussian window function at $r$ on the tangent plane $T_{r}$ as

$$
g_{r}\left(x_{r}, y_{r}\right)=c \exp \left\{-\frac{\left(x_{r}^{2}+y_{r}^{2}\right)}{\sigma^{2}}\right\} .
$$

$$
\begin{aligned}
u(\boldsymbol{x}) \approx & \sum_{m} \sum_{n} \sum_{k} \sum_{l} a_{m n k l} h_{z}(x-m X, y-n Y) \\
& \times c \sigma^{2} \pi \exp \left\{-\pi^{2} \sigma^{2}\left[\left(\frac{x-m X}{\lambda \sqrt{(x-m X)^{2}+(y-n Y)^{2}+z^{2}}}-k F_{x}\right)^{2}+\left(\frac{y-n Y}{\lambda \sqrt{(x-m X)^{2}+(y-n Y)^{2}+z^{2}}}-l F_{y}\right)^{2}\right]\right\} \\
& \times \exp \left\{-j 2 \pi\left[\left(\frac{x-m X}{\lambda \sqrt{(x-m X)^{2}+(y-n Y)^{2}+z^{2}}}-k F_{x}\right) m X+\left(\frac{y Y}{\lambda \sqrt{(x-m X)^{2}+(y-n Y)^{2}+z^{2}}}-l F_{y}\right) n Y\right]\right\},
\end{aligned}
$$

where the discrete analysis coefficients $a_{m n k l}$ are found by using Eq. (8).

\section{B. Decomposition for the Curved Input Surface Case}

The expression given by Eq. (15) calculates the 3D diffraction field by an integration over the local Gaussian beams for a given input field on a planar surface. Now, let us consider a continuously differentiable curved surface $S \subset \mathbb{R}^{3}$. We represent the points on $S$ by the vector $r \in \mathbb{R}^{3}$.

In order to define modulated Gaussian window functions on $S$ let us first define the local coordinate systems on the curved surface. We define the local coordinate system $\boldsymbol{x}_{\boldsymbol{r}}=$ $\left(x_{r}, y_{r}, z_{r}\right)$ at $r$ such that $x_{r}$ and $y_{r}$ axes are orthogonal to each other and they are placed on the tangent plane $T_{r}$ to $S$ at $r$. The $z_{r}$ axis is chosen to give a right-handed orthogonal coordinate system (see Fig. 1). Please note that $S$ is assumed to be an orientable surface. For all regions of $S$ where $u(r)$ is effective, we assume that $S$ is smooth enough such that it can be treated as locally planar within the support of the Gaussian window functions. Here, we define the "support" casually to describe the domain such that the Gaussian window function is practically zero outside of that domain. The smoothness
The modulated Gaussian window function with modulation frequencies of $f_{x_{r}}$ and $f_{y_{r}}$ is then given as

$$
\begin{aligned}
& g_{r}\left(x_{r}, y_{r}\right) \exp \left\{j 2 \pi\left(f_{x_{r}} x_{r}+f_{y_{r}} y_{r}\right)\right\} \\
& \quad=c \exp \left\{-\frac{\left(x_{r}^{2}+y_{r}^{2}\right)}{\sigma^{2}}\right\} \exp \left\{j 2 \pi\left(f_{x_{r}} x_{r}+f_{y_{r}} y_{r}\right)\right\} .
\end{aligned}
$$

Now, let us define the neighborhood $V_{T_{r}}$ of the point $\boldsymbol{r}$ on $T_{\boldsymbol{r}}$ such that the Gaussian window function is effectively zero outside of $V_{T_{r}}$. Then we define the neighborhood $V_{S_{r}}$ of $r$ on $S$ such that its orthogonal projection onto $T_{r}$ is $V_{T_{r}}$ and represent the corresponding projected field on $V_{T_{r}}$ as $u_{r}\left(x_{r}, y_{r}\right)$. The smoothness assumption of $S$ together with the small extent of the Gaussian window function ensures that the projected field $u_{r}\left(x_{r}, y_{r}\right)$ represents the field $u(r)$ on $V_{S_{r}}$ with a negligible error. Using the projected field $u_{r}\left(x_{r}, y_{r}\right)$ we find the coefficients of the modulated Gaussian window functions at $\boldsymbol{r}$ by the analysis equation, defined on the tangent plane $T_{r}$, as 


$$
\begin{aligned}
a\left(\boldsymbol{r}, f_{x_{r}}, f_{y_{r}}\right)= & \int_{-\infty}^{\infty} \int_{-\infty}^{\infty} u_{r}\left(x_{r}, y_{r}\right) g_{r}^{*}\left(x_{r}, y_{r}\right) \\
& \times \exp \left\{-j 2 \pi\left(f_{x_{r}} x_{r}+f_{y_{r}} y_{r}\right)\right\} \mathrm{d} x_{r} \mathrm{~d} y_{r} \\
\approx & \iint_{V_{T_{r}}} u_{r}\left(x_{r}, y_{r}\right) g_{r}^{*}\left(x_{r}, y_{r}\right) \\
& \times \exp \left\{-j 2 \pi\left(f_{x_{r}} x_{r}+f_{y_{r}} y_{r}\right)\right\} \mathrm{d} x_{r} \mathrm{~d} y_{r} .
\end{aligned}
$$

In order to calculate the diffraction field in $3 \mathrm{D}$ space due to the field $u(r)$ on $S$, we need to propagate the Gaussian beams corresponding to the modulated Gaussian window functions on $S$. Before doing this, let us relate the representation of a vector $\boldsymbol{x}$ in $(x, y, z)$ global coordinate system to its representation $x_{r}$ in $\left(x_{r}, y_{r}, z_{r}\right)$ local coordinate system as

$$
x_{r}=R_{r} x-r
$$

Here, $\boldsymbol{R}_{\boldsymbol{r}}$ is the $3 \times 3$ rotation matrix. Note that both the local coordinate system $\left(x_{r}, y_{r}, z_{r}\right)$ and $\boldsymbol{R}_{r}$ change with position $\boldsymbol{r}$ on $S$. We gave the expression for the propagation of a Gaussian beam corresponding to a shifted and modulated Gaussian window function on a planar surface in Eq. (14). Now, using the definition given in Subsection 3.A, we define $g_{h}\left(\boldsymbol{x}, f_{x}, f_{y}\right)$ as the $3 \mathrm{D}$ field at the observation point $\boldsymbol{x}=$ $[x, y, z]^{T}$ on a plane parallel to $z=0$ plane, due to a modulated Gaussian window function at $(x, y)=(0,0)$ on $z=0$ plane, with the modulation frequencies $f_{x}$ and $f_{y}$ along the $x$ and $y$ axes, respectively. Remember that $(x, y, z)$ is the global coordinate system as shown in Fig. 1. By using the expression derived in Subsection $\underline{3 . \mathrm{A}}$ and given by Eq. ( $\underline{14}), g_{h}\left(\boldsymbol{x}, f_{x}, f_{y}\right)$ is written as

$$
\begin{aligned}
g_{h}\left(\boldsymbol{x}, f_{x}, f_{y}\right) \approx & h_{z}(x, y) c \sigma^{2} \pi \\
& \times \exp \left\{-\pi^{2} \sigma^{2}\left[\left(\frac{x}{\lambda|\boldsymbol{x}|}-f_{x}\right)^{2}+\left(\frac{y}{\lambda|\boldsymbol{x}|}-f_{y}\right)^{2}\right]\right\} .
\end{aligned}
$$

At this point we should note that $g_{h}\left(x, f_{x}, f_{y}\right)$ is defined for the case of parallel input and observation planes because the underlying RS diffraction model gives the field relation between two parallel planes. Therefore, a reference plane (even it is hypothetical) is needed to specify a $3 \mathrm{D}$ field. The $2 \mathrm{D}$ field on the infinite extent reference plane uniquely determines the corresponding 3D field, provided that the propagation direction components of the waves along the $z$ axis is always positive. The $2 \mathrm{D}$ fields over the planes that are parallel to

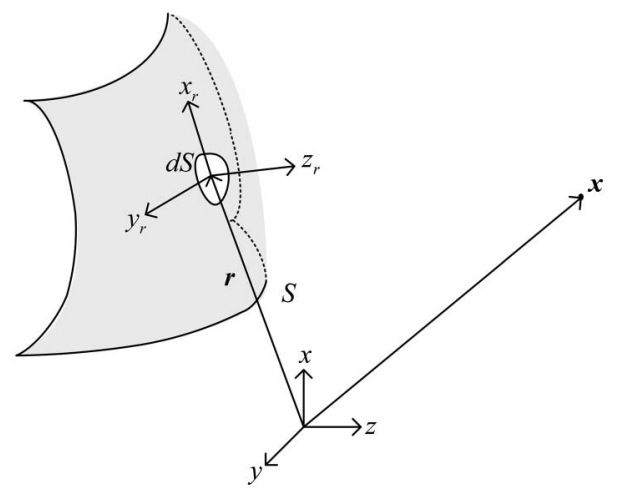

Fig. 1. Local coordinate system on the input curved surface. the reference plane can be found by using the RS diffraction model. What we call the 3D field is the concatenation of such $2 \mathrm{D}$ fields over the planes at different depths. Therefore, the field $u(r)$ given on $S$ represents a 2D field resulting from the intersection of such a 3D field by the curved surface $S$. With these definitions, the meaning of a $3 \mathrm{D}$ field, given a $2 \mathrm{D}$ field on a curved surface $S$, should be correctly interpreted: the $3 \mathrm{D}$ field at a point gives the scalar field on a plane that passes through that point and parallel to the reference plane. Note that the extent of $S$ along the reference plane is assumed to be infinite such that the projection of $S$ onto the reference plane covers the entire plane. Therefore, under the assumption that the propagation direction components of the waves along the $z$ axis is restricted to be positive, the 3D field is uniquely determined by the 2D field on $S$.

We use the expression given by Eq. (21), with proper coordinate transformations, for the propagation of the Gaussian beams induced by the curved surface $S$. Hence, for a given field $u(\boldsymbol{r})$ on $S$ we find the diffraction field at $\boldsymbol{x}$ by an integration over all Gaussian beams as

$$
\begin{aligned}
u(x) \approx & \iint_{S} \iint_{y} a\left(\boldsymbol{r}, f_{x_{r}}, f_{y_{r}}\right) g_{h}\left(\boldsymbol{x}_{\boldsymbol{r}}, f_{x_{r}}, f_{y_{r}}\right) \mathrm{d} f_{x_{r}} \mathrm{~d} f_{y_{r}} \mathrm{~d} S \\
\approx & \iint_{S} \iint_{S} a\left(\boldsymbol{r}, f_{x_{r}}, f_{y_{r}}\right) h_{z_{r}}\left(x_{\boldsymbol{r}}, y_{\boldsymbol{r}}\right) c \sigma^{2} \pi \\
& \times \exp \left\{-\pi^{2} \sigma^{2}\left[\left(\frac{x_{\boldsymbol{r}}}{\lambda\left|\boldsymbol{x}_{\boldsymbol{r}}\right|}-f_{x_{r}}\right)^{2}+\left(\frac{y_{\boldsymbol{r}}}{\lambda\left|\boldsymbol{x}_{\boldsymbol{r}}\right|}-f_{y_{r}}\right)^{2}\right]\right\} \\
& \times \mathrm{d} f_{x_{r}} \mathrm{~d} f_{y_{r}} \mathrm{~d} S,
\end{aligned}
$$

where $\boldsymbol{x}_{\boldsymbol{r}}=\boldsymbol{R}_{\boldsymbol{r}} \boldsymbol{x}-\boldsymbol{r}$, as given by Eq. (20).

The continuous signal decomposition given by Eq. (22) is extremely redundant. By choosing the discrete set of evaluation points on $S$ and shift steps in frequency properly, we can still decompose the field on $S$ into a discrete set of modulated Gaussian window functions on the tangent planes of $S$. That is, it is possible to write $u(x)$ as a sum of Gaussian beams that correspond to Gaussian window functions at discrete positions $\left\{\boldsymbol{r}_{1}, \boldsymbol{r}_{2}, \ldots, \boldsymbol{r}_{n}\right\}$ on $S$ and having discrete modulation frequencies as multiples of $F_{x}$ and $F_{y}$, as

$$
\begin{aligned}
u(\boldsymbol{x}) \approx & \sum_{i=1}^{n} \sum_{k} \sum_{l} a_{\boldsymbol{r}_{i} k l} g_{h}\left(\boldsymbol{x}_{\boldsymbol{r}_{i}}, k F_{x}, l F_{y}\right) \\
\approx & \sum_{i=1}^{n} \sum_{k} \sum_{l} a_{\mathbf{r}_{i} k l} h_{z_{\mathbf{r}_{i}}}\left(x_{\boldsymbol{r}_{i}}, y_{\boldsymbol{r}_{i}}\right) c \sigma^{2} \pi \\
& \times \exp \left\{-\pi^{2} \sigma^{2}\left[\left(\frac{x_{\boldsymbol{r}_{i}}}{\lambda\left|\boldsymbol{x}_{\boldsymbol{r}_{i}}\right|}-k F_{x}\right)^{2}+\left(\frac{y_{\boldsymbol{r}_{i}}}{\lambda\left|\boldsymbol{x}_{\boldsymbol{r}_{i}}\right|}-l F_{y}\right)^{2}\right]\right\},
\end{aligned}
$$

where

$$
\begin{aligned}
a_{\boldsymbol{r}_{i} k l}= & \int_{-\infty}^{\infty} \int_{-\infty}^{\infty} u_{\boldsymbol{r}_{i}}\left(x_{\boldsymbol{r}_{i}}, y_{\boldsymbol{r}_{i}}\right) w_{\boldsymbol{r}_{i}}^{*}\left(x_{\boldsymbol{r}_{i}}, y_{\boldsymbol{r}_{i}}\right) \\
& \times \exp \left\{-j 2 \pi\left(k F_{x} x_{\boldsymbol{r}_{i}}+l F_{y} y_{\boldsymbol{r}_{i}}\right)\right\} \mathrm{d} x_{\boldsymbol{r}_{i}} \mathrm{~d} y_{\boldsymbol{r}_{i}} \\
\approx & \iint_{V_{T_{\boldsymbol{r}_{i}}}} u_{\boldsymbol{r}_{i}}\left(x_{\boldsymbol{r}_{i}}, y_{\boldsymbol{r}_{i}}\right) w_{\boldsymbol{r}_{i}}^{*}\left(x_{\boldsymbol{r}_{i}}, y_{\boldsymbol{r}_{i}}\right) \\
& \times \exp \left\{-j 2 \pi\left(k F_{x} x_{\boldsymbol{r}_{i}}+l F_{y} y_{\boldsymbol{r}_{i}}\right)\right\} \mathrm{d} x_{\boldsymbol{r}_{i}} \mathrm{~d} y_{\boldsymbol{r}_{i}} .
\end{aligned}
$$


If the discrete evaluation points are taken on a regular grid, then, similar to the planar surface case, an analysis window function $w_{r}\left(x_{r}, y_{r}\right)$, which is valid for all $\boldsymbol{r}_{i} \in\left\{\boldsymbol{r}_{1}, \boldsymbol{r}_{2}, \ldots, \boldsymbol{r}_{n}\right\}$, can be found [26]. However, because it is usually not possible to place a regular grid on the entire surface, the surface can be partitioned into small patches and each patch can be treated separately. The locality of the Gaussian synthesis window function ensures the applicability of such a partitioning. Note that in the discrete case, $V_{T_{r}}$ is used to represent the support of the analysis window function $w_{r}\left(x_{r}, y_{r}\right)$ on $T_{r}$. For the applicability of Eq. (24), the analysis window function should be sufficiently narrow such that within its support the surface can be treated as locally planar. By a sufficiently dense sampling in space and frequency, it is possible to have an analysis window function that is as narrow as the synthesis window function [23].

The accuracy of Eq. (22) mainly depends on three factors. The first factor is the adaption of an approximate 3D Gaussian beam expression [see Eq. (15)]. As mentioned in Subsection 3.A, the accuracy of the approximate 3D Gaussian beam expression $g_{h}\left(\boldsymbol{x}, f_{x}, f_{y}\right)$ is better for a narrower Gaussian window function. The second factor is the application of the developed Gaussian beam expression to curved surfaces, even though it is developed for a planar surface. A narrower Gaussian window function is also desired to decrease the error resulting from the application of such an expression to a curved surface, because for a narrower Gaussian window function, the locally planar assumption of the given curved surface becomes more reasonable. Finally, the last factor is the independent treatment of mutually coupled patches on the curved surface. Here, we use the word "patch" to refer to a surface element on the curved surface that represents the support of the Gaussian window function written on it. If a beam induced by a patch illuminates another disjoint patch on the curved surface, then such patches cannot be treated independently [16]. We call such patches mutually coupled patches. Independent treatment of mutually coupled patches produces inconsistent 3D field solutions, because a beam induced by one of the mutually coupled patches alters the field value on the other patch [16]. Because a narrower Gaussian window function results in Gaussian beams having larger angular spreads, the likelihood that such a wider Gaussian beam illuminates another patch on the surface increases. Therefore, as opposed to first two factors given above, a narrower Gaussian window function produces worse results in terms of mutual couplings.

In order to show the effect of the width of the Gaussian window function on the mutual couplings, two scenarios are illustrated in Fig. 2. $S_{1}$ and $S_{2}$ patches shown in Fig. 2(a) are not mutually coupled, because the beam induced by $S_{1}$ does not illuminate $S_{2}$, and vice versa. In Fig. 2(b) we decrease the width of the Gaussian window function on $S_{1}$ patch. Therefore, angular spread of $B_{1}$ increases, and $S_{2}$ is now illuminated by also $B_{1}$. Thus, $S_{1}$ and $S_{2}$ patches become mutually coupled. Note that as we continue to decrease the width of the Gaussian window functions on the curved surface, the angular spreads of the corresponding beams become larger and larger.

In addition to the locality of the Gaussian window functions, mutual couplings also depend on the structure of the input curved surface, as well. As an example, mutual coupling

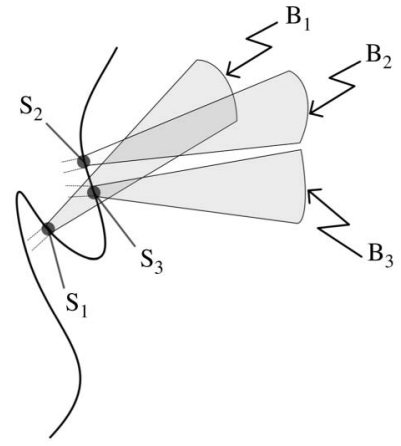

(a)

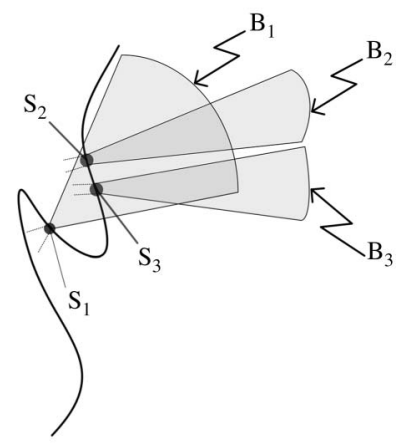

(b)
Fig. 2. Mutual couplings between patches on a curved surface (2D cross sections of the surface and beams are shown for the sake of simplicity). (a) Mutual coupling between $S_{1}$ and $S_{3}$ patches, (b) mutual coupling between $S_{1}$ and $S_{2}$ patches (in addition to the mutual coupling between $S_{1}$ and $S_{3}$ patches).

between $S_{1}$ and $S_{3}$ patches on a curved surface is shown in Figs. 2(a) and 2(b). Because of the structure of the curved surface, the mutual coupling between these two patches cannot be reduced to negligible levels, even if we change the width of the Gaussian window function on $S_{1}$. In this study, we restrict the set of curved surfaces to a feasible set for which it is possible to keep the mutual couplings at negligible levels so that the developed formulations are applicable.

Gaussian beams having a large angular spread are not desirable also because of associated computational reasons. Such beams illuminate a larger volume of space compared to the narrower beams, and, therefore, a higher number of them become effective (superpose) at a given observation point; this brings an extra computational burden in the computation of Eq. (22).

All these discussions lead us to choose a Gaussian window function having a sufficiently small width, such that the accuracy of the derived formulations are satisfactory in field calculations, but nevertheless, the computational complexity is still in tolerable limits and the mutual couplings are negligible.

\section{SIMULATION RESULTS}

Simulations are presented for the $2 \mathrm{D}(x, z)$ space, for the sake of simplicity. Note that the $z$ variable still represents the depth. We aim to find the $2 \mathrm{D}$ diffraction field due to a field specified on a curved line (instead of a curved surface in 3D space) by using the proposed Gaussian beam decomposition method.

In order to write the signal space as a sum of finite number of plane waves (whose propagation direction components along the $y$ axis is zero), we assume a periodicity along the $x$ axis. The $2 \mathrm{D}$ cross sections of such plane waves by the $(x, z)$ plane are used in $(x, z)$ space. The periodicity, together with the discretization of the functions, enable us to calculate the coefficients of the Gaussian beams by using the discrete Fourier transform (DFT) [see Eq. (26)]. An example simulation setup is presented in Fig. 3. As shown in the figure, the curved line is periodic along the $x$ axis with period $X_{p}$. The $2 \mathrm{D}(x, \boldsymbol{z})$ field is also periodic along the $x$ axis with the same period. Therefore, the signal on the periodic curved line, $\tilde{u}(\boldsymbol{r}(l))$, which is obtained by intersecting such a periodic $2 \mathrm{D}$ field, is also periodic. Note that we represent the signals on 


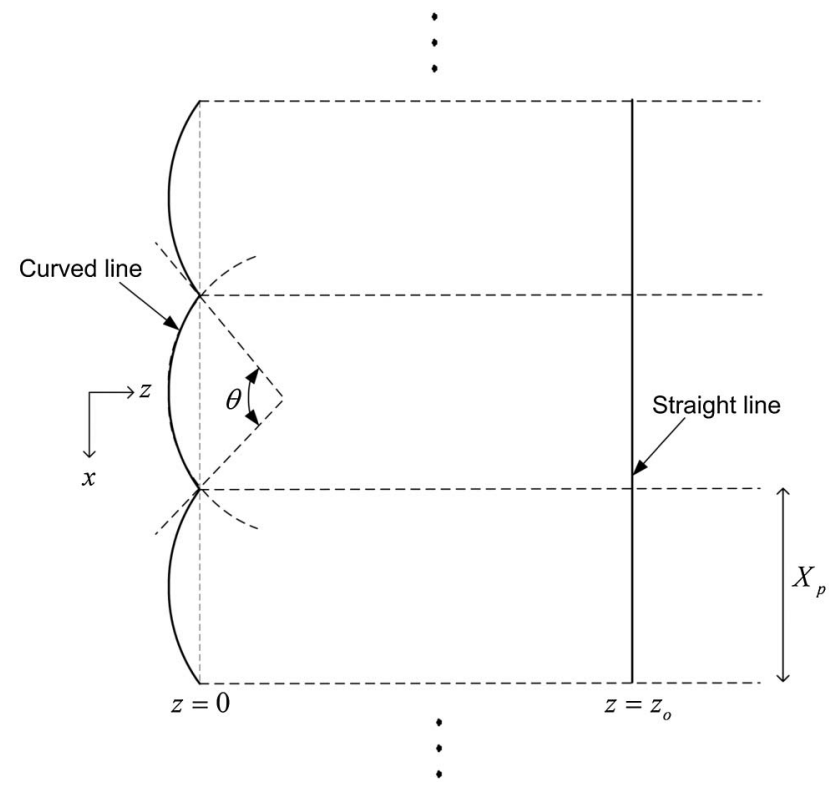

Fig. 3. 2D simulation setup, which is periodic along the $x$ axis (the fields used in the simulations repeat themselves between each dashed horizontal line shown in the figure).

the curved line, with respect to the $2 \mathrm{D}(x, z)$ global coordinate system, by using the arc-length parameterization $\boldsymbol{r}(l)$. Also note that we use the tilde sign to denote periodic signals on the curved line and we represent one period of such signals without the tilde sign.

The discrete and periodic input signal $\tilde{u}_{d}[n]$ is obtained by uniformly sampling the continuous signal $\tilde{u}(\mathbf{r}(l))$ with the sampling step $L_{s}$; i.e., $\tilde{u}_{d}[n]=\tilde{u}\left(\boldsymbol{r}\left(L_{s} n\right)\right) . N$ samples per period are obtained after discretization. The finite-length Gaussian synthesis window function $g(r(l))$ is sampled with the same sampling step and periodically extended over the periodic curved line to have the discrete and periodic synthesis window function $\tilde{g}_{d}[n]$. The total number of Gaussian beams to be included in the diffraction field calculation depends on the choice of discrete shift steps in the position and modulation frequency of the Gaussian synthesis window function. The shift steps in $n$ and the normalized frequency domain $k$ are denoted as $M$ samples and $1 / K$ cycles/sample, respectively. The corresponding total number of Gaussian beams used in the decomposition is therefore equal to $N K / M$. The ratio $K / M$ also affects the shape of the analysis window function corresponding to $\tilde{g}_{d}[n]$. For the case of oversampling of the space-frequency domain $K / M>1$, the expansion coefficients are dependent, because the set of modulated and shifted Gaussian windows is overcomplete. Therefore, the analysis window function corresponding to the synthesis window function is not unique. In the simulations we choose $M$ and $K$ such that $K / M=2$, which corresponds to oversampling by a factor of 2 . For this case, we find the analysis window function $\tilde{w}_{d}[n]$ corresponding to the specified Gaussian synthesis window function by using the relation that gives the minimum $L_{2}$-norm solution, developed in [23]. The parameter of the Gaussian synthesis window function, $\sigma$, is chosen as $2.20 \mu \mathrm{m}$. One period of the resulting discrete synthesis window function, $g_{d}[n]=\exp \left\{-L_{s}^{2} n^{2} / \sigma^{2}\right\}$ for $L_{s}=$ $0.26 \mu \mathrm{m}$, and the corresponding discrete analysis window function, $w_{d}[n]$, are shown in Figs. $\underline{4}$ and $\underline{5}$, respectively, for $n=-64,-63, \ldots, 63$.

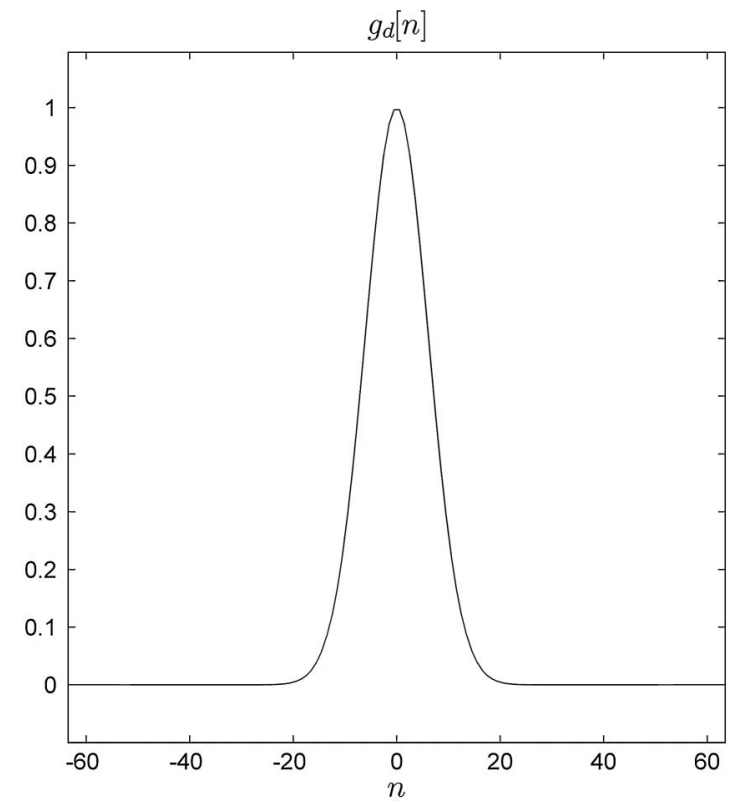

Fig. 4. Discrete Gaussian synthesis window function $g_{d}[n]=$ $\exp \left\{-L_{s}^{2} n^{2} / \sigma^{2}\right\}$ for $\sigma=2.20 \mu \mathrm{m}$ and $L_{s}=0.26 \mu \mathrm{m}$.

Note that we presented the formulations in Subsection 3.B by using the tangent planes of the curved surface, for the $\overline{3 \mathrm{D}}$ case. However, in the $2 \mathrm{D}$ simulations, we do the calculations on the curved line with the signals defined above (instead of the tangent lines of the curved line), for the sake of clarity. The curved lines that we work with are almost locally planar within the supports of the analysis and synthesis window functions. Therefore, such an approach is appropriate to simulate the developed formulations in $2 \mathrm{D}(x, z)$ space. Regardless of a dimension reduction, we omit the constant multipliers and simply assume them as unity during the computations. For a given periodic input signal $\tilde{u}_{d}[n]$, the coefficients of

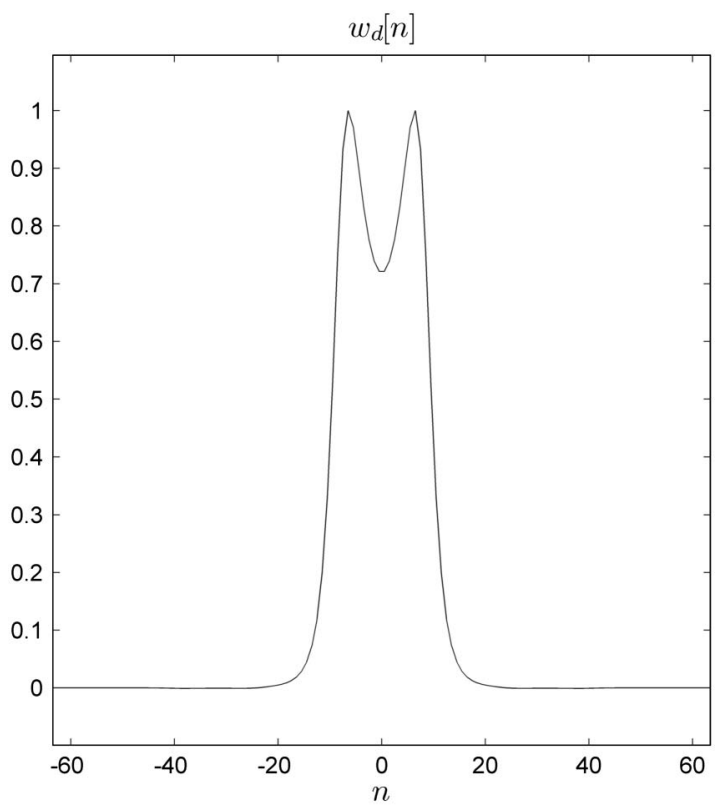

Fig. 5. Discrete analysis window function corresponding to the Gaussian synthesis window function $g_{d}[n]=\exp \left\{-L_{s}^{2} n^{2} / \sigma^{2}\right\}$, for $\sigma=$ $2.20 \mu \mathrm{m}$ and $L_{s}=0.26 \mu \mathrm{m}$, for the case that the shift parameters in space and frequency are $M=16$ and $K=32$, respectively. 
the modulated Gaussian window functions on the curved line are found by the discrete analysis equation as

$$
\begin{aligned}
a_{m k} & =\sum_{n=\frac{-N}{2}}^{\frac{N}{2}-1} \tilde{u}_{d}[n] \tilde{w}_{d}^{*}[n-m M] \exp \left(-j 2 \pi \frac{k}{K} n\right), \\
m & \in\left\{\frac{-N}{2 M}, \frac{-N}{2 M}+1, \ldots, \frac{N}{2 M}-1\right\}, \\
k & \in\left\{\frac{-K}{2}, \frac{-K}{2}+1, \ldots, \frac{K}{2}-1\right\},
\end{aligned}
$$

where $N, M$, and $K$ are positive integer valued ( $N$ is an even integer) simulation parameters that are defined above. Equation (25) can be rewritten as

$$
\begin{aligned}
a_{m k}= & \sum_{n_{1}=\frac{-K}{2}}^{\frac{K}{2}-1} \sum_{n_{2}=\frac{-N}{2 K}}^{\frac{N}{2 K}-1} \tilde{u}_{d}\left[n_{1}+n_{2} K\right] \tilde{w}_{d}^{*}\left[n_{1}+n_{2} K-m M\right] \\
& \times \exp \left(-j 2 \pi \frac{k}{K} n_{1}\right), \\
m & \in\left\{\frac{-N}{2 M}, \frac{-N}{2 M}+1, \ldots, \frac{N}{2 M}-1\right\}, \\
k & \in\left\{\frac{-K}{2}, \frac{-K}{2}+1, \ldots, \frac{K}{2}-1\right\} .
\end{aligned}
$$

Thus, the analysis coefficients are found by computing a $K$-point DFT of the signal

$$
\tilde{u}_{w_{d}}\left[n_{1}\right]=\sum_{n_{2}=\frac{-N}{2 K}}^{\frac{N}{2 K}-1} \tilde{u}_{d}\left[n_{1}+n_{2} K\right] \tilde{w}_{d}^{*}\left[n_{1}+n_{2} K-m M\right]
$$

(which is periodic with period $K$ ) for each fixed $m$. Please note that even though some of the indicated $(m, k)$ pairs correspond to Gaussian beams having propagation directions with negative components along the $z$ axis, the coefficients of such beams are zero. The reason is that we restrict the $2 \mathrm{D}$ field to superposition of propagating waves whose propagation direction components along the $z$ axis are positive.

As a consequence of the periodicity together with the discretization of the signals on the periodic curved line, for each $(m, k)$ pair, Eq. (25) gives the coefficient of the corresponding parallel Gaussian beams induced by the curved line (see Fig. 6). Because the Gaussian beams are local, only a few of these parallel duplicates are effective at a given observation point. We label the windows that are taken into account, for the $(m, k)$ pair, as the set of integers $\left\{b_{m k}, b_{m k}+1, \ldots, b_{m k}+c\right\}$. Here, the window label $b_{m k}$ refers to the region of the $2 \mathrm{D}$ space with $b_{m k} X_{p}-\frac{X_{p}}{2} \leq x<b_{m k} X_{p}+\frac{X_{p}}{2}$, taking the center window $\left(\frac{X_{p}}{2} \leq x<\frac{X_{p}}{2}\right)$ as a reference. For example, while calculating the field at $\left(x_{0}, z_{0}\right)$ due to the parallel beams shown in Fig. $\underline{6}$, only the windows with labels 1 and 2 (that induce $B_{1}$ and $\bar{B}_{2}$ beams, respectively) are taken into account. Under the above definitions, we find the field at $(x, z)$ due to the periodic input curved line by using the following equation as

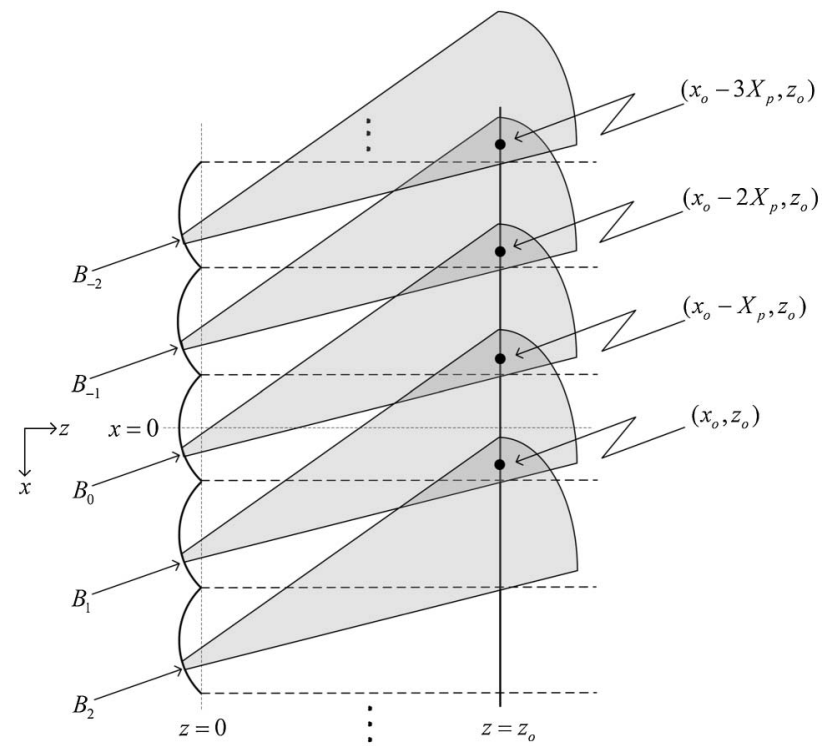

Fig. 6. Periodic Gaussian beams induced by the periodic pattern over the periodic curved line.

$$
\begin{aligned}
& u(x, z) \\
& \approx \sum_{m=\frac{-N}{2 M}}^{\frac{N}{2 M}-1} \sum_{k=\frac{-K}{2}}^{\frac{K}{2}-1} \sum_{b \in S_{w}} a_{m k} g_{h}\left(\boldsymbol{R}_{\boldsymbol{r}\left(m M L_{s}\right)}\left[x-b X_{p}, z\right]^{T}-\boldsymbol{r}\left(m M L_{s}\right), \frac{k}{K L_{s}}\right) \\
& S_{w}=\left\{b_{m k}, b_{m k}+1, \ldots, b_{m k}+c\right\}
\end{aligned}
$$

where $\boldsymbol{R}_{\boldsymbol{r}\left(m M L_{s}\right)}$ is the $2 \times 2$ rotation matrix relating the $2 \mathrm{D}$ $(x, z)$ global coordinate system and the local coordinate system (one axis is along the tangent line and the other axis is along the normal line of the curved line) at $\boldsymbol{r}\left(m M L_{s}\right)$.

A circular arc with a measure of $\theta=30^{\circ}$ is used as one period of the input curved line (see Fig. 3 ). The period along the $x$ axis, $X_{p}$, is chosen as $0.26 \mathrm{~mm}$. We uniformly sample the signal on the curved line with a sampling step $L_{s}=0.51 \lambda$ resulting in $N=1024$ samples in one period of the signal. Note that $\lambda$, which is taken as $500 \mathrm{~nm}$, is the wavelength of the monochromatic light. We take $M=16$ and $K=32$, resulting in 2048 different parallel Gaussian beams. Thus, the oversampling factor is 2 .

As an illustration of the decomposition procedure on the curved line, three discrete sinc functions, shifted to different positions, are written on the circular arcs. One period, $u_{d}[n]$, of this signal is shown in Fig. 7. One period of the diffraction field due to the input signal $\tilde{u}_{d}[n]$ is given in Fig. 8 . This field is found by superposing various Gaussian beam components that correspond to modulated Gaussian window functions on the circular segments with different $m$ and $k$ parameters. An example Gaussian beam component, which corresponds to the modulated Gaussian window function with parameters $m=16$ and $k=1$, is presented in Fig. $\underline{9}$. Note that Figs. $\underline{8}$ and $\underline{9}$ are obtained by sampling the $u(x, \bar{z})$ field with a sampling step $X_{s}=0.5 \lambda$ along both axes. Also note that the beams shown in Figs. 8 and 9 are propagating from $z=-\infty$ to $z=$ $+\infty$ and their beam waists are taken on the curved line.

For a more general case, we start with specifying a 2D $(x, z)$ field by randomly choosing the coefficients of the finite number of plane waves whose frequency components along the $x$ axis occupy the $\left[\frac{-0.70}{\lambda}, \frac{0.70}{\lambda}\right]$ cycles $/ m$ frequency band. Then we 


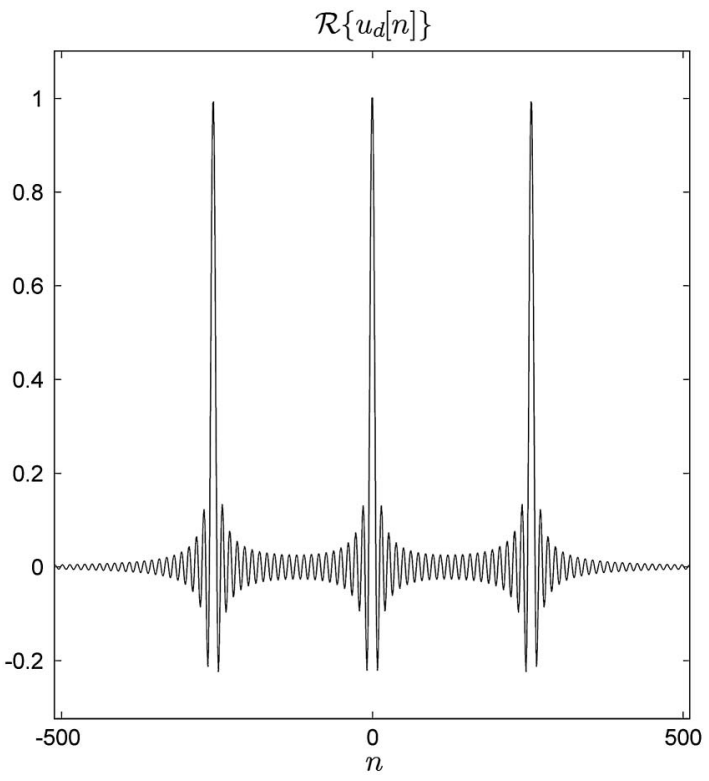

Fig. 7. Real part of the discrete input signal, $u_{d}[n]$, on the circular arc with a measure of $30^{\circ}$ (imaginary part is zero).

intersect this test field by the curved line. Because the developed formulations are not applicable for the nonsmooth regions of the curved line, we window the resulting field so that the field values around the edges of the curved line (nonsmooth regions) are set to zero (see Fig. 10). We first write the field on the curved line as a sum of modulated and shifted Gaussian window functions and then find the coefficients of each Gaussian elementary signal according to Eq. (26). Afterward, we calculate the samples of the diffraction field on the observation line at $z=0.1 \mathrm{~m}$ via Eq. (27). Note that this distance is sufficiently large for the usage of the developed Gaussian beam expression, given by Eq. (14), in accurate field calculations. In order to test the proposed approach, we

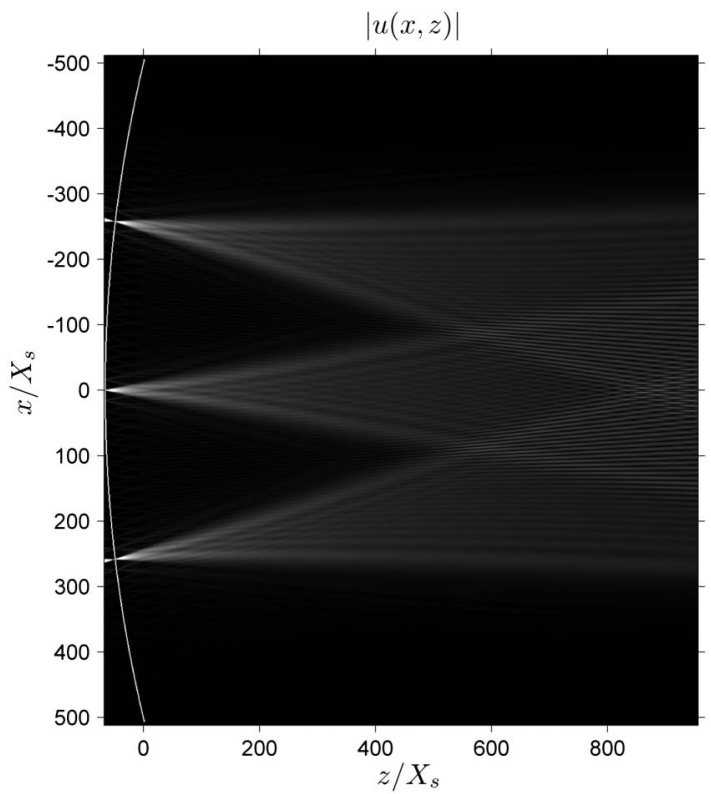

Fig. 8. Magnitude of the 2D diffraction field due to the input signal $\tilde{u}_{d}[n]$, one period of which is shown in Fig. 7 , on the circular arc with a measure of $30^{\circ}$.

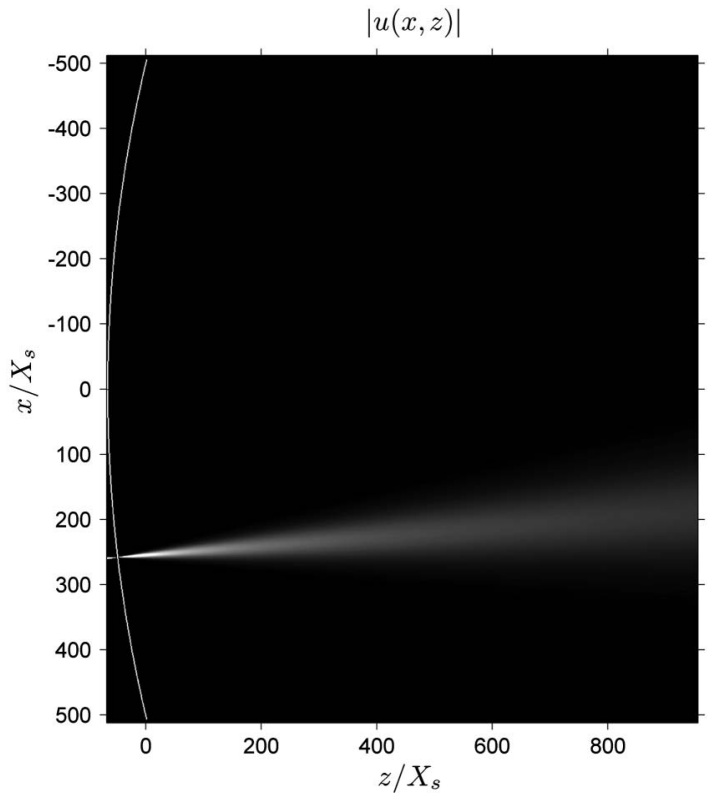

Fig. 9. Magnitude of the 2D diffraction field due to a single shifted and modulated Gaussian window function, with parameters $m=16$ and $k=1$, on the circular arc with a measure of $30^{\circ}$.

first use the PWD method, given in Section 2 , to find the 2D continuous field due to the discrete and periodic signal computed on the observation line. The periodic continuous field due to the discrete and periodic signal $u\left(n X_{s}, z_{o}\right)$, observed at $z=z_{o}$, is computed by the 2D PWD as

$$
u(x, z)=\frac{1}{N} \sum_{k=\frac{-N}{2}}^{\frac{N}{2}-1} A_{k} \exp \left\{j 2 \pi\left(\frac{k}{X_{s} N} x+\sqrt{\frac{1}{\lambda^{2}}-\frac{k^{2}}{X_{s}^{2} N^{2}}}\left(z-z_{o}\right)\right)\right\},
$$

where
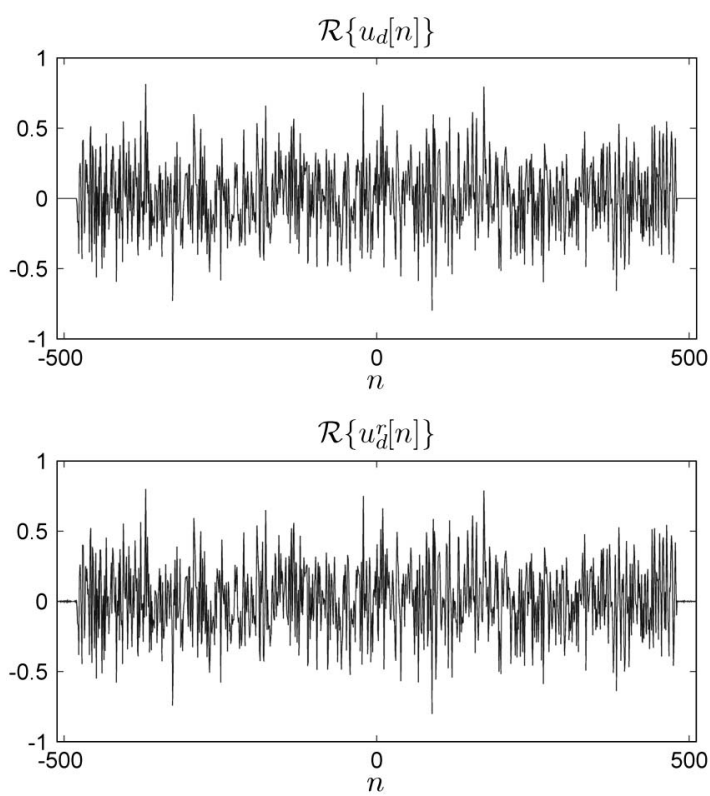

Fig. 10. Real parts of the original, $u_{d}[n]$, and reconstructed, $u_{d}^{r}[n]$, signals on the circular arc with a measure of $30^{\circ}$. 


$$
\begin{gathered}
A_{k}=\sum_{n=\frac{-N}{2}}^{\frac{N}{2}-1} u\left(n X_{s}, z_{o}\right) \exp \left(-j 2 \pi \frac{k n}{N}\right), \\
k \in\left\{\frac{-N}{2}, \frac{-N}{2}+1, \ldots, \frac{N}{2}-1\right\},
\end{gathered}
$$

where, as mentioned before, $X_{s}$ is the sampling step along the $x$ axis. Note that because of the discretization and the periodicity along the $x$ axis, we find only the coefficients of 1024 [total number of samples in one period of $u\left(x, z_{0}\right)$ ] plane waves by using 1024-point DFT of the sampled field at $z=z_{0}$. In order to find the reconstruction error, we resample the continuous field obtained by Eq. (28) at the discrete points on the curved line and compare the resulting discrete reconstructed signal with the original discrete test signal written on the curved line. One periods of the real parts of the original and reconstructed signals are shown in Fig. 10 (the imaginary parts have similar plots). We are able to reconstruct the field on the periodic circular segments with a normalized error [see Eq. (12)] in the order of $10^{-4}$. This shows that the developed formulations work well in computing the diffraction field in $(x, z)$ space for a field specified on the given periodic circular arcs. Similar results are obtained for many other curved lines that satisfy the smoothness assumption and for which the mutual couplings between the disjoint patches on it are negligible for a specified Gaussian window function. Note that for the circular arcs that we use, the mutual couplings between the disjoint patches inducing the Gaussian beams are at negligible levels.

\section{CONCLUSIONS}

We present a local signal decomposition method to calculate the 3D diffraction field due to a field specified on a curved surface. The field given on the curved surface is decomposed into a sum of shifted and modulated Gaussian window functions. Then the 3D diffraction field is calculated as a sum of local Gaussian beams each of which corresponding to a modulated Gaussian window function on the curved surface. Satisfactory results are obtained by using the given approximate Gaussian beam expression.

The angular spread of a local Gaussian beam is considerably lower than the angular spread of a 3D field induced by a point source. Thus, the mutual couplings between the point sources on a curved surface are significantly higher than the mutual couplings between the patches that induce local Gaussian beams. Therefore, the proposed signal decomposition method provides a model with better accuracy compared to the source model approaches [ $\underline{8}-14]$ for the problem of finding the $3 \mathrm{D}$ field due to a field specified on a smooth curved surface.

The field model introduced in [16] (which is also explained in Section 2) provides an exact solution for the problem defined in this study. That is an important development over the source model approaches [8-14]. However, the computational complexity of the exact field model given in [16] increases significantly for large sizes of surfaces, because the defined problem is desired to be solved by an inverse problem approach. Thus, in the discrete case a large matrix inversion should be performed; this is a computationally highly demanding operation for a meaningful size of surface. Moreover, because the intersections of infinite extent plane waves by the given curved surface are needed to be stored as a part of the algorithm proposed in [16], a memory problem occurs for large sizes of surfaces. On the other hand, our method is suitable for parallel programming to reduce the computation time considerably, because we find the 3D field as a sum of Gaussian beams each of which can be calculated separately, in a parallel fashion. Furthermore, the proposed method requires much less memory. That is, although our method does not produce exact field solutions as the model given in [16], it provides quite accurate results and applicable to even large size surfaces.

The choice of the width of the Gaussian window function used in the decomposition is based on a trade-off. The developed approximate Gaussian beam expression gives better results as the width of the Gaussian window function is reduced. Such a narrower Gaussian window function is also desired in order to satisfy the smoothness constraint of a given surface for the applicability of the developed formulations. On the other hand, Gaussian beams corresponding to narrower Gaussian window functions have larger angular spreads. Therefore, the likelihood, that the patches on the curved surface (inducing such beams) become mutually coupled, increases. Moreover, because a higher number of such beams should be taken into account (compared to the case where Gaussian beams having smaller angular spreads are used) while calculating the diffraction field at a given observation point, the computational burden also increases. We conclude that the width of the Gaussian window function should be chosen sufficiently small such that the derived formulations produce satisfactory results in field calculations, but the mutual couplings are still negligible and the computational complexity is still in tolerable limits. For some surfaces, such a choice may not exist. The proposed method produces quite accurate results with a tolerable complexity for surfaces for which it is possible to find such a width for the Gaussian window function.

\section{ACKNOWLEDGMENTS}

Erdem Şahin acknowledges the partial support of TÜBİTAK for this work in the form of a scholarship. We also thank Erdem Ulusoy for fruitful discussions.

\section{REFERENCES}

1. L. Onural, F. Yaraş, and H. Kang, "Digital holographic threedimensional video displays," Proc. IEEE 99, 576-589 (2011).

2. N. Sato, H. Aritake, M. Kato, M. Ishimoto, and M. Nakashima, "Stereoscopic display apparatus," U.S. patent 5,594,559 (14 January 1997).

3. J. Hahn, H. Kim, Y. Lim, G. Park, and B. Lee, "Wide viewing angle dynamic holographic stereogram with a curved array of spatial light modulators," Opt. Express 16, 12372-12386 (2008).

4. W. J. Dallas, "Computer-generated holograms," in Digital Holography and Three-Dimensional Display, T. C. Poon, ed. (Springer, 2006), pp. 1-49.

5. C. Slinger, C. Cameron, and M. Stanley, "Computer-generated holography as a generic display technology," Computer 38, 46-53 (2005).

6. J. W. Goodman, Introduction to Fourier Optics (McGraw-Hill, 1996), 2nd ed.

7. J. D. Gaskill, "The propagation and diffraction of optical wave fields," in Linear Systems, Fourier Transforms, and Optics (Wiley, 1978), Chap. 10.

8. J. Waters, "Holographic image synthesis utilizing theoretical methods," Appl. Phys. Lett. 9, 405-407 (1966). 
9. T. Yatagai, "Stereoscopic approach to 3-D display using computer-generated holograms," Appl. Opt. 15, 2722-2729 (1976).

10. K. Matsushima and M. Takai, "Recurrence formulas for fast creation of synthetic three-dimensional holograms," Appl. Opt. 39, 6587-6594 (2000).

11. M. Lucente, "Optimization of hologram computation for realtime display," Proc. SPIE 1667, 32-43 (1992).

12. K. Matsushima, "Computer-generated holograms for threedimensional surface objects with shade and texture," Appl. Opt. 44, 4607-4614 (2005).

13. M. Janda, I. Hanák, and L. Onural, "Hologram synthesis for photorealistic reconstruction," J. Opt. Soc. Am. A 25, 3083-3096 (2008)

14. L. Ahrenberg, "Methods for transform, analysis and rendering of complete light representations," Ph.D. thesis (Max-PlanckInstitut für Informatik, 2010).

15. G. C. Sherman, "Application of the convolution theorem to Rayleigh's integral formulas," J. Opt. Soc. Am. 57, 546-547 (1967).

16. G. B. Esmer, "Calculation of scalar optical diffraction field from its distributed samples over the space," Ph.D. thesis (Bilkent University, 2010)

17. G. B. Esmer, L. Onural, and H. M. Ozaktas, "Exact diffraction calculation from fields specified over arbitrary curved surfaces," Opt. Commun. 284, 5537-5548 (2011).
18. D. Gabor, "Theory of communication," J. IEE 93, 429-457 (1946).

19. M. J. Bastiaans, "Gabor's signal expansion and the Zak transform,” Appl. Opt. 33, 5241-5255 (1994).

20. M. J. Bastiaans, "Expansion of an optical signal into a discrete set of Gaussian beams," Optik 57, 95-102 (1980).

21. L. Onural, "Exact solution for scalar diffraction between tilted and translated planes using impulse functions over a surface," J. Opt. Soc. Am. A 28, 290-295 (2011).

22. P. Flandrin, Time-Frequency/Time-Scale Analysis (Academic, 1999).

23. M. J. Bastiaans, "Oversampling in Gabor's signal expansion by an integer factor," in Proceedings of the IEEE-SP International Symposium on Time-Frequency and Time-Scale Analysis, 1994 (IEEE, 1994), pp. 280-283.

24. A. Janssen, "Gabor representation of generalized functions," J. Math. Anal. Appl. 83, 377-394 (1981)

25. K. Tang, Mathematical Methods for Engineers and Scientists Fourier Analysis, Partial Differential Equations and Variational Models (Springer, 2007).

26. M. J. Bastiaans and A. J. van Leest, "Gabor's signal expansion and the Gabor transform based on a non-orthogonal sampling geometry," in Sixth International Symposium on Signal Processing and Its Applications, 2001 (IEEE, 2001), pp. 162-163. 